\title{
Balanço energético e pegada de carbono nos sistemas de produção integrada e convencional de feijão-comum irrigado
}

\author{
Heliton Fernandes do Carmo ${ }^{(1)}$, Beáta Emöke Madari(2), Alcido Elenor Wander(2), \\ Flavia Rabelo Barbosa Moreira(2), Augusto Cesar de Oliveira Gonzaga ${ }^{(2)}$, Pedro Marques da Silveira(2), \\ Aluisio Goulart Silva ${ }^{(2)}$, José Geraldo da Silva(2) e Pedro Luiz Oliveira de Almeida Machado(2)
}

\begin{abstract}
(1)Universidade Federal de Goiás, Departamento de Solos, Rodovia GO-462, Km 0, Campus II, CEP 74001-970 Goiânia, GO, Brasil. E-mail: heliton15@hotmail.com (2)Embrapa Arroz e Feijão, Rodovia GO-462, Km 12, CEP 75375-000 Santo Antônio de Goiás, GO, Brasil. E-mail: beata.madari@embrapa.br,alcido.wander@embrapa.br,flavia.barbosa@embrapa.br,augusto.gonzaga@embrapa.br,pedro.silveira@embrapa.br; aluisio.silva@embrapa.br, josegeraldo.silva@embrapa.br, pedro.machado@embrapa.br
\end{abstract}

Resumo - O objetivo deste trabalho foi avaliar a contribuição do sistema de produção integrada de feijão-comum (PI), em comparação ao sistema de produção convencional, quanto ao uso de energia, à eficiência energética e à pegada de carbono, contabilizados desde a fabricação de insumos até a colheita de grãos nas fazendas, tendo-se excluído o transporte. Foram selecionadas quatro fazendas em Cristalina, GO, na terceira época de produção de feijão, em 2009. O sistema PI reduziu o uso médio de energia em 3,1\%, em comparação ao convencional. Os resultados variaram de 22.759,9 a 25.518,4 $\mathrm{MJ} \mathrm{ha}^{-1}$. A adubação nitrogenada com 6.584,0 $\mathrm{MJ}^{-1} \mathrm{ha}^{-1}$, as operações mecanizadas com 5.309,4 $\mathrm{MJ} \mathrm{ha}^{-1} \mathrm{e}$ a irrigação com 4.961,4 $\mathrm{MJ} \mathrm{ha}^{-1}$ tiveram a maior participação quanto ao uso de energia. A eficiência energética em PI $(2,16)$ foi superior à observada no sistema convencional $(2,01)$. Não houve diferença significativa entre os dois sistemas quanto à pegada de carbono por hectare, mas a pegada de carbono por quilograma de feijão produzido no PI $\left(0,301 \mathrm{~kg} \mathrm{CO}_{2}\right.$-eq $\left.\mathrm{kg}^{-1}\right)$ foi significativamente menor do que no convencional $\left(0,325 \mathrm{~kg} \mathrm{CO}_{2}\right.$-eq kg$\left.{ }^{-1}\right)$. A melhora da eficiência da adubação nitrogenada, por meio do uso de fixação biológica de $\mathrm{N}$, e a racionalização da mecanização são os componentes dos sistemas de produção que mais contribuem para a diminuição da pegada de carbono.

Termos para indexação: Phaseolus vulgaris, adubação mineral nitrogenada, análise de ciclo de vida, eficiência energética, pivô central.

\section{Energy balance and carbon footprint in the conventional and integrated production systems of irrigated common bean}

\begin{abstract}
The objective of this work was to evaluate the contribution of an integrated farming system (PI) of common bean, in comparison with convectional farming, as to energy use, energy efficiency, and carbon footprint, considered from input manufacturing to grain harvest in the farms, excluding transport. Four farms were selected in Cristalina, state of Goiás, Brazil, during the third harvest season of common bean, in 2009. The PI system reduced the average energy use by $3.1 \%$, in comparison with the conventional system. Values ranged from 22,759.9 to 25,518.4 $\mathrm{Mg} \mathrm{ha}^{-1}$. Mineral $\mathrm{N}$ fertilization with 6,584.0 $\mathrm{MJ} \mathrm{ha}^{-1}$, agricultural operations with 5,309.4 $\mathrm{MJ} \mathrm{ha}^{-1}$, and irrigation with 4,961.4 $\mathrm{MJ} \mathrm{ha}^{-1}$ had the largest participation in energy use. Energy efficiency in PI (2.16) was higher than in the conventional system (2.01). There was no significant difference between the two systems as to carbon footprint per hectare, but carbon footprint per kilogram of yielded bean in PI $\left(0.301 \mathrm{~kg} \mathrm{CO}_{2}\right.$-eq kg-1) was significantly lower than in conventional system $\left(0.325 \mathrm{~kg} \mathrm{CO}_{2}\right.$-eq kg-1 $)$. The improvement of $\mathrm{N}$ fertilizer use efficiency by $\mathrm{N}$ biological fixation and the rationalization of machinery are the production system components that contributes the most for the reduction of carbon footprint.
\end{abstract}

Index terms: Phaseolus vulgaris L, mineral $\mathrm{N}$ fertilization, life cycle analysis, energy efficiency, Center pivot irrigation.

\section{Introdução}

O cultivo de grãos pode ser visto como um sistema que incorpora a produção de materiais ricos em energia, por meio da fotossíntese e uso de insumos altamente dependentes de combustíveis fósseis como diesel, fertilizantes e pesticidas (Pimentel \& Pimentel, 2006). Em estudo recente, sobre o balanço energético de sistemas de produção de grãos no Cerrado, Sá 
et al. (2013) constataram maior balanço energético na produção de biodiesel de milho do que na da soja. Em um contexto de sustentabilidade, o uso de energia é fator essencial para o desenvolvimento econômico e, também, em razão do uso de insumos dependentes de combustível fóssil, a causa de problemas ambientais como o aquecimento global pela emissão de gases de efeito estufa (Cowell \& Parkinson, 2003). Consequentemente, além do balanço energético, surgiu a necessidade da determinação das emissões totais de gases de efeito estufa (GEEs) associadas à produção agrícola. A pegada de carbono representa essa determinação e é uma das métricas (categorias de impacto) da avaliação do ciclo de vida do sistema de produção agrícola (Rotz et al., 2010). No Brasil, a determinação da pegada de carbono é comum em outros setores, como a construção civil (Passuello et al., 2014), e é uma medida da quantidade total de dióxido de carbono $\left(\mathrm{CO}_{2}\right)$ ou equivalente de $\mathrm{CO}_{2}$ gerado por uma determinada atividade (Wiedmann \& Minx, 2007).

A população brasileira tem-se mobilizado na busca por alimentos seguros, que não ofereçam perigo à sua saúde, de maneira similar às exigências mercadológicas internacionais (Portocarrero \& Kososki, 2009). Surgiu, assim, a produção integrada agropecuária (PI Brasil) ou produção integrada (PI), articulada pelo Ministério da Agricultura, Pecuária e Abastecimento (Mapa). A produção integrada teve início na Europa, no início dos anos 1970 (Boller et al., 1998) e, segundo Morris \& Winter (1999), ela se caracterizou como alternativa à agricultura embasada no monocultivo, sob intensa aplicação de insumos sintéticos, mas sem as rigorosas exigências da agricultura orgânica certificada.

Em avaliação realizada em propriedades agrícolas da região de Cristalina, GO, embora o PI Brasil de feijão-comum tenha apresentado desempenho econômico mais favorável do que o sistema convencional (Silva et al., 2012), não se tem ainda uma avaliação criteriosa da sustentabilidade ambiental dos dois tipos de sistema de produção.

O objetivo deste trabalho foi avaliar a contribuição do sistema de produção integrada de feijão-comum (PI), em comparação ao sistema de produção convencional, quanto ao uso de energia, eficiência energética e pegada de carbono, contabilizados desde a fabricação de insumos até à colheita de grãos nas fazendas, tendo-se excluído o transporte.

\section{Material e Métodos}

As práticas agrícolas da região de Cristalina, GO, comumente adotadas para a produção de feijão de terceira época, são: adequação da área (reforma de terraços, ajustes do pivô central e calagem), semeadura com adubação fertilizante mineral, proteção de plantas, adubação de cobertura e colheita. Similarmente ao trabalho conduzido por Abeliotis et al. (2013), o foco do presente estudo ficou restrito à produção de feijão, e não ao armazenamento, à distribuição ou ao consumo. Assim, foram incluídos todos os estágios da produção dos insumos, como fabricação de fertilizantes e pesticidas, e aplicação à lavoura (Figura 1), tendo-se excluído o transporte.

O estudo foi realizado em 2009, em quatro fazendas na região de Cristalina, GO, tradicional produtora de feijão-comum, à terceira época de plantio. Todas as quatro fazendas avaliadas adotam a semeadura direta, mas não o sistema plantio direto, por não haver rotação de culturas com plantas de cobertura, para o manejo da palha à superfície do solo. O tipo de solo é o Latossolo Vermelho-Amarelo, e as características gerais das áreas de estudo nas fazendas estão descritas na Tabela 1 . Exceto na Fazenda 1, cujo solo muito argiloso (669 $\left.\mathrm{g} \mathrm{kg}^{-1}\right)$ apresenta acidez fraca $\left(\mathrm{pH}_{\text {água }} 6,9\right)$ e médio teor de bases trocáveis, o solo das Fazendas 2, 3 e 4 vão de argiloso a muito argiloso (469 a $689 \mathrm{~g} \mathrm{~kg}^{-1}$ ), com acidez média $\left(\mathrm{pH}_{\text {água }} 5,3\right.$ a 5,9) e baixos teores de bases trocáveis. Todos os solos apresentam teor médio, de 26 a $32 \mathrm{~g} \mathrm{dm}^{-3}$, de matéria orgânica.

Para a semeadura, utilizaram-se espaçamentos de 45 ou $50 \mathrm{~cm}$ entre fileiras, com 8 a 10 sementes por metro. Para o PI, seguiram-se rigorosamente as recomendações de Paula Junior et al. (2008), com especial atenção para o manejo integrado de pragas, adubação nitrogenada de cobertura, com uso de clorofilômetro, e ausência de aplicação foliar de nutrientes ou condicionadores. O manejo da irrigação foi igual para os dois sistemas, com $30 \mathrm{~cm}$ de lâmina aplicada durante o ciclo da cultura, por meio de irrigâmetro (Silveira et al., 2009). Conforme Sá et al. (2013), para a obtenção do balanço energético, utilizou-se a razão entre a energia produzida pelo sistema (saída) e a energia investida para a obtenção dos produtos agrícolas (entrada). $\mathrm{Na}$ irrigação dos dois sistemas de cultivo, consideraram-se os mesmos valores de consumo energético (Pimentel \& Patzek, 2005) e emissão de gás de efeito estufa (West \& Marland, 2002). Os quatro pivôs centrais das fazendas 
eram movidos à eletricidade gerada em hidroelétrica. Os valores estimados para a emissão de GEEs foram embasados na hipótese de os pivôs centrais serem movidos à eletricidade gerada por motor a diesel, que é utilizado por cerca de $30 \%$ dos irrigantes no Brasil (Oliveira \& Zocoler, 2010). As estimativas de emissão de GEEs, no presente estudo, têm como base a quantidade de energia fóssil requerida para a síntese e o processamento desses insumos, numa abordagem de análise de ciclo de vida (ACV), conforme sugerido por Raucci et al. (2015), quanto ao cultivo da soja em Mato Grosso.

A colheita foi feita de forma semimecanizada ou mecanizada, com arranquio manual das plantas ou corte e enleiramento mecanizado (ceifadora), e recolhimento e trilha mecanizados com recolhedora-trilhadora ou com máquina automotriz (Silva et al., 2012).

A unidade funcional selecionada foi a produção de 1 kg de grão de feijão-comum em PI e no sistema convencional, numa safra de outono-inverno. $\mathrm{O}$ valor adotado para a composição energética do grão de

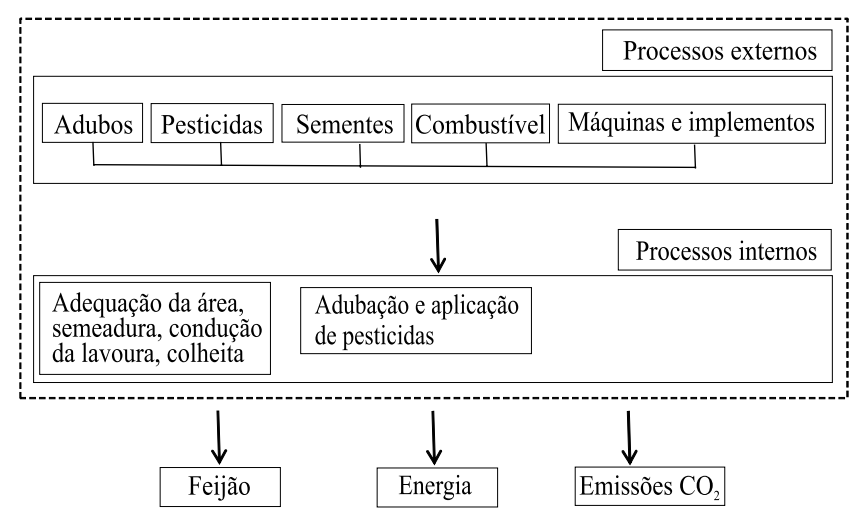

Figura 1. Descrição da abrangência dos processos (fronteiras do estudo) e principais insumos utilizados na produção de feijão-comum de terceira época (adaptado de Abeliotis et al., 2013; Raucci et al., 2015). feijão cru foi $14,65 \mathrm{MJ} \mathrm{kg}^{-1}$, conforme Bassinello (2009). Os valores referentes ao uso de energia, em quilocaloria (kcal) ou megajoule (MJ) de cada insumo ou equipamento utilizado, foi estimado para o cultivo em 1 ha, e a eficiência energética foi estimada em relação à produção de feijão em 1 ha, ou seja, a relação entre a energia gerada (produtividade de grãos de feijão) e o total de energia utilizada para produzir grãos de feijão por hectare, conforme sugerido por Camargo et al. (2013).

Para a determinação do total de energia usada na produção, considerou-se a energia usada na produção de fertilizantes, pesticidas, diesel, máquinas, implementos, eletricidade e sementes similarmente ao procedimento conduzido por Helander \& Delin (2004).

O levantamento dos principais insumos (processos externos) utilizados para o cultivo de feijão, à terceira época, considerou-se a demanda energética e a emissão de gases de efeito estufa (Tabela 2) na fabricação de adubos fertilizantes (Mello, 1989; West \& Marland, 2002; Williams et al., 2006), o calcário (Pimentel \& Patzek, 2005), os pesticidas (Audsley et al., 2009), o óleo diesel (West \& Marland, 2002; Pimentel \& Patzek, 2005; Soares et al., 2009) e as sementes (Quadros \& Kokuszka, 2007). Em alguns casos, foi possível obter valores específicos para os ingredientes ativos de pesticidas utilizados, porém, utilizou-se um valor médio, quando não foi possível se obter informação para determinado ingrediente ativo.

Em razão da baixa quantidade de resíduos de plantas $\left(<800 \mathrm{~kg}\right.$ matéria seca ha $\left.{ }^{-1}\right)$, por ocasião da semeadura de feijão, não se consideraram as emissões referentes à cobertura morta. Embora utilizados na maioria das fazendas sob sistema convencional, também não se consideraram a energia usada e as emissões - referentes a ingressos denominados promotores de crescimento ou formulações nutricionais organominerais (ex. Nitrogram, BR Organ) -, pela inexistência de informação e baixa quantidade proporcional utilizada $(0,47$ a $3,12 \%$

Tabela 1. Características gerais das áreas de estudo com cultivo de feijão, em sistema de produção integrada (PI) e convencional, na região de Cristalina, Goiás, na terceira época de produção, em 2009.

\begin{tabular}{|c|c|c|c|c|c|}
\hline \multirow[t]{2}{*}{ Área de estudo } & \multicolumn{2}{|c|}{ Área (ha) } & \multicolumn{2}{|c|}{ Cultivar - Produtividade $\left(\mathrm{kg} \mathrm{ha}^{-1}\right)$} & \multirow[t]{2}{*}{ Cultura antecedente } \\
\hline & Produção integrada & Convencional & Produção integrada & Convencional & \\
\hline Fazenda 1 & 40 & 40 & Pérola -3.465 & Pérola -3.015 & Milho (Zea mays) \\
\hline Fazenda 2 & 20 & 20 & Pérola -3.470 & Pérola - 3.435 & Milho (Zea mays) \\
\hline Fazenda 3 & 21 & 64 & Pérola - 3.432 & Pérola - 3.511 & Capim-colchão(D. ciliaris $)$ \\
\hline Fazenda 4 & 25 & 75 & Valente -3.456 & Valente -3.320 & Urochloa ruziziensis \\
\hline
\end{tabular}


de participação). Em nenhum local foi feita a inoculação de bactérias fixadoras de nitrogênio nas sementes.

Os valores referentes à emissão dos principais gases de efeito estufa $\left(\mathrm{CO}_{2}, \mathrm{~N}_{2} \mathrm{O}\right.$ e $\left.\mathrm{CH}_{4}\right)$, durante a fabricação, aplicação e emissão direta, foram expressos em equivalente de $\mathrm{CO}_{2}\left(\mathrm{CO}_{2}\right.$-eq $)$. Para a conversão das emissões em $\mathrm{CO}_{2}$-eq, adotaram-se os seguintes valores: 21, para o potencial de aquecimento do metano; e 310 ,

Tabela 2. Demanda de energia e emissão de gases de efeito estufa (GEE), na composição ou fabricação de insumos utilizados na produção de feijão-comum de terceira época.

\begin{tabular}{lrr}
\hline Insumos & Emissão de GEE & Energia dispendida \\
\hline Mão de obra ${ }^{(1)}$ & $0,026 \mathrm{~kg} \mathrm{ha}^{-1}$ & $300 \mathrm{kcal} \mathrm{h}^{-1}$ \\
Calcário & $0,036 \mathrm{~kg} \mathrm{~kg}^{-1}$ & $281 \mathrm{kcal} \mathrm{kg}^{-1}$ \\
Óleo Diesel das operações ${ }^{(2)}$ & $5,06 \mathrm{~kg} \mathrm{l}^{-1}$ & $11.400 \mathrm{kcal} \mathrm{L}^{-1}$ \\
Fertilizante nitrogenado (N) & $0,86 \mathrm{~kg} \mathrm{~kg}^{-1}$ & $14.977 \mathrm{kcal} \mathrm{kg}^{-1}$ \\
Fertilizante fosfatado $\left(\mathrm{P}_{2} \mathrm{O}_{5}\right)$ & $0,16 \mathrm{~kg} \mathrm{~kg}^{-1}$ & $3.384 \mathrm{kcal} \mathrm{kg}^{-1}$ \\
Fertilizando potássico $\left(\mathrm{K}_{2} \mathrm{O}\right)$ & $0,12 \mathrm{~kg} \mathrm{~kg}^{-1}$ & $2.268 \mathrm{kcal} \mathrm{kg}^{-1}$ \\
Sementes de feijão(3) & $0,69 \mathrm{~kg} \mathrm{~kg}^{-3}$ & $8.320 \mathrm{kcal} \mathrm{kg}^{-1}$ \\
Pesticidas & \\
Herbicidas & & \\
Inseticidas & $18,25 \mathrm{~kg} \mathrm{~kg}^{-1}$ i.a & $63.055 \mathrm{kcal} \mathrm{kg}^{-1}$ i.a. \\
Fungicidas & $14,79 \mathrm{~kg} \mathrm{~kg}^{-1}$ i.a. & $51.113 \mathrm{kcal} \mathrm{kg}^{-1}$ i.a. \\
Herbicida (2,4-D) & $11,93 \mathrm{~kg} \mathrm{~kg}^{-1}$ i.a. & $41.320 \mathrm{kcal} \mathrm{kg}^{-1}$ i.a. \\
Herbicida (paraquat) & $6,00 \mathrm{~kg} \mathrm{~kg}^{-1}$ i.a. & $20.779 \mathrm{kcal} \mathrm{kg}^{-1}$ i.a. \\
Herbicida (diuron) & $31,67 \mathrm{~kg} \mathrm{~kg}^{-1}$ i.a. & $109.630 \mathrm{kcal} \mathrm{kg}^{-1}$ i.a. \\
Herbicida (diquat) & $18,56 \mathrm{~kg} \mathrm{~kg}^{-1}$ i.a. & $64.249 \mathrm{kcal} \mathrm{kg}^{-1}$ i.a. \\
Herbicida (glifosato) & $27,60 \mathrm{~kg} \mathrm{~kg}^{-1}$ i.a. & $95.538 \mathrm{kcal} \mathrm{kg}^{-1}$ i.a. \\
Fungicida (piraclostrobina) & $37,60 \mathrm{~kg} \mathrm{~kg}^{-1}$ i.a. & $108.436 \mathrm{kcal} \mathrm{kg}^{-1}$ i.a. \\
Fungicida (fluazinam) & $48,43 \mathrm{~kg} \mathrm{~kg}^{-1}$ i.a. & $167.670 \mathrm{kcal} \mathrm{kg}^{-1}$ i.a. \\
Fungicida (tebuconazol) & $40,98 \mathrm{~kg} \mathrm{~kg}^{-1}$ i.a. & $141.874 \mathrm{kcal} \mathrm{kg}^{-1}$ i.a. \\
Fungicida (carbendazim) & $38,02 \mathrm{~kg} \mathrm{~kg}^{-1}$ i.a. & $131.604 \mathrm{kcal} \mathrm{kg}^{-1}$ i.a. \\
Irrigação & $28,29 \mathrm{~kg} \mathrm{~kg}^{-1}$ i.a. & $97.927 \mathrm{kcal} \mathrm{kg}^{-1}$ i.a. \\
\hline & $5,25 \mathrm{~kg} \mathrm{~cm}^{-1}$ & $39.500 \mathrm{kcal} \mathrm{cm}^{-1}$ \\
\hline
\end{tabular}

${ }^{(1)} \mathrm{O}$ gasto energético da mão de obra, segundo Souza et al. (2008), é 300 $\mathrm{kcal}$ h ou seja, $1,257 \mathrm{MJ} \mathrm{h}^{-1}$. Para West \& Marland (2002) a mão de obra tem um gasto de $0,020 \mathrm{~kg} \mathrm{CO}_{2}$-eq/MJ. Assim, o gasto por hora é de $0,026 \mathrm{~kg}$ $\mathrm{CO}_{2}$-eq. (2) Para West \& Marland (2002), na produção de diesel, há emissão de $0,02195 \mathrm{~kg} \mathrm{CO}_{2}$-eq MJ ${ }^{-1}$. Segundo Pimentel \& Patzek (2005), para a produção do combustível, são gastos $11.400 \mathrm{kcal} \mathrm{L}^{-1}$ ou $47,65 \mathrm{MJ} \mathrm{L}^{-1}$, que equivale, então, a $1,05 \mathrm{~kg} \mathrm{CO}_{2}$-eq L ${ }^{-1}$ de diesel. Segundo Soares et al. (2009), na combustão completa de $1 \mathrm{~L}$ de diesel, são produzidos $4,01 \mathrm{~kg} \mathrm{CO} \mathrm{CO}_{2}$-eq. Assim, considera-se a soma do $\mathrm{CO}_{2}$ emitido na fabricação e o emitido na queima, o que gera 5,06 $\mathrm{kg} \mathrm{CO}_{2}$-eq L $\mathrm{L}^{-1}$ de diesel ou $229,6 \mathrm{MJ} \mathrm{L}^{-1}$. Não se considerou a emissão de $\mathrm{CO}_{2}$-eq para o transporte de diesel até a fazenda. ${ }^{(3)}$ Considerou-se que a produção de $1,0 \mathrm{~kg}$ de sementes exige 1,0 MJ, para gerar emissão de $0,0197 \mathrm{~kg}$ de $\mathrm{CO}_{2}$-eq (West \& Marland, 2002). ${ }^{(4)}$ Valores médios para pesticidas, no caso da inexistência de valor específico para o ingrediente ativo utilizado. Para transformar $\mathrm{MJ} \mathrm{em} \mathrm{CO}_{2}$-eq L $\mathrm{L}^{-1}$ de i.a. (ingrediente ativo), utilizou-se o fator 0,069 (Audsley, 2009). ${ }^{(5)}$ Considerou-se o uso de $4,6 \mathrm{kWh}$, para aplicar $1 \mathrm{~mm}$ de água $\left(10 \mathrm{~m}^{3} \mathrm{ha}^{-1}\right)$, conforme Marouelli \& Silva (2011), ou $860 \mathrm{kcal}$ para cada $1 \mathrm{kWh}$ utilizado, conforme West \& Marland (2002). Adotando-se o valor de 5,06 $\mathrm{kg} \mathrm{CO}_{2}$-eq L $\mathrm{eq}^{-1}$ de diesel utilizado, conforme Soares et al. (2009), tem-se 1,04 L de diesel para 5,25 $\mathrm{kg} \mathrm{CO}_{2}$-eq. Para o presente estudo, adotou-se que $1 \mathrm{~L}$ de diesel é necessário para aplicar $1 \mathrm{~cm}$ de água e gerar $5,25 \mathrm{~kg} \mathrm{CO}_{2}$-eq. para o óxido nitroso, preconizado por Solomon et al. (2007).

A demanda energética e a emissão de gases de efeito estufa foram contabilizadas para a mão de obra empregada no tratamento de sementes, monitoramento de pragas e doenças, nas áreas de PI e na colheita dos dois sistemas de produção (Souza et al., 2008).

O cálculo do consumo de diesel (sem biodiesel), nas operações mecanizadas, incluiu o tipo de máquina e o implemento representativo para a produção de feijão-comum na região, conforme descrito em Silva et al. (2012) e sugestão de Grisso et al. (2010) sobre o consumo de combustível por hectare.

Para determinar a quantidade de energia fóssil, embutida na fabricação de máquinas e equipamentos normalmente utilizados na produção de feijão-comum, à terceira época, calculou-se a demanda específica de energia (DEE), conforme proposto por Ulbanere \& Ferreira (1989), com subsequente estimativa da pegada de carbono ( $\mathrm{kg} \mathrm{CO}_{2}$-eq ha $\left.{ }^{-1}\right)$ da DEE.

Especificamente para o uso de energia, contabilizaram-se todos os insumos e operações envolvidos na produção de feijão, nos dois sistemas de cultivo, inclusive aqueles que foram adotados somente em uma fazenda ou sistema de cultivo (como colheita mecanizada, colheita manual e recolhimento/trilha), ou aquele que foi igual em todas as fazendas e sistemas de cultivo (como irrigação).

Os dados foram submetidos à análise de componentes principais, por meio do programa The Unscrambler $\mathrm{X} 10.2$ (Camo Software Inc.), para separar as quatro fazendas nos sistemas convencional e PI, com base nas variáveis quantificadas. A comparação das médias das variáveis referentes ao uso de energia, pegada de carbono por hectare e por quilograma de grão de feijão produzido, e as médias das estimativas da eficiência energética, para cada tipo de sistema de cultivo foram comparadas pelo erro padrão da média.

\section{Resultados e Discussão}

A energia total utilizada por hectare na produção de feijão, nos dois sistemas de cultivo, para cada fazenda, variou de 22.759,9 $\mathrm{MJ} \mathrm{ha}^{-1}$ a $24.449,8 \mathrm{MJ}^{-1} \mathrm{ha}^{-1}$ no PI e de $23.373,8 \mathrm{MJ} \mathrm{ha}^{-1}$ a $25.518,4 \mathrm{MJ} \mathrm{ha}^{-1}$ no convencional (Tabelas 3 e 4).

A redução no uso médio de energia por hectare no PI foi de $3,1 \%$, em relação ao sistema convencional, ou o equivalente a 3,3 $\mathrm{L} \mathrm{ha}^{-1}$ de diesel consumido, para uma 
conversão de 229,6 $\mathrm{MJ} \mathrm{L}^{-1}$. Bailey et al. (2003) avaliaram o uso de energia na produção convencional e na produção integrada, em diferentes localidades na Grã-Bretanha, na sucessão de cultivos de trigo-pousio-ervilha e batata, ou colza- trigo-pousio, e observaram a economia de $8 \%$ no uso de energia na produção integrada. No entanto, Alluvione et al. (2011), em experimentos de longo prazo (>14 anos) na região de Turim, Norte da Itália, observaram economia de 17,5, 26,3 e 19\%, no uso de energia no PI, em comparação à produção convencional

Tabela 3. Consumo e eficiência (balanço) no uso de energia (MJ), na produção convencional e integrada (PI) de feijão-comum (Phaseolus vulgaris), na terceira época, nas fazendas 1 e 2, em Cristalina, GO.

\begin{tabular}{|c|c|c|c|c|c|c|c|c|c|c|c|c|}
\hline \multirow[t]{3}{*}{ Ingresso } & \multicolumn{6}{|c|}{ Fazenda 1} & \multicolumn{6}{|c|}{ Fazenda 2} \\
\hline & \multicolumn{3}{|c|}{ Convencional } & \multicolumn{3}{|c|}{ PI } & \multicolumn{3}{|c|}{ Convencional } & \multicolumn{3}{|c|}{ PI } \\
\hline & Quantidade & Energia & $(\%)$ & Quantidade & Energia & $(\%)$ & Quantidade & Energia & $\overline{(\%)}$ & Quantidade & Energia & $(\%)$ \\
\hline Calcário (kg) & $1.500,0$ & $1.764,7$ & 7,3 & $1.500,0$ & $1.764,7$ & 7,7 & $1.500,0$ & $2.647,1$ & 10,4 & $1.500,0$ & $1.764,7$ & 7,2 \\
\hline $\mathrm{N}(\mathrm{kg})$ & 105,0 & $6.584,0$ & 27,2 & 89,5 & $5.612,1$ & 24,4 & 99,11 & $6.214,8$ & 24,4 & 99,0 & $6.207,9$ & 25,4 \\
\hline $\mathrm{P}_{2} \mathrm{O}_{5}(\mathrm{~kg})$ & 75,4 & $1.067,9$ & 4,4 & 117,0 & $1.657,7$ & 7,2 & 87,6 & $1.241,1$ & 4,9 & 87,0 & $1.232,6$ & 5,0 \\
\hline $\mathrm{K}_{2} \mathrm{O}(\mathrm{kg})$ & 36,0 & 341,8 & 1,4 & 24,0 & 227,9 & 1,0 & 87 & 826,1 & 3,2 & 87,0 & 826,1 & 3,4 \\
\hline Herbicida (kg) & 3,0 & 923,1 & 3,8 & 2,8 & 946,0 & 4,1 & 1,98 & 706,8 & 2,8 & 2,0 & 706,8 & 2,9 \\
\hline Fungicida (kg) & 2,5 & 429,3 & 1,8 & 0,5 & 87,2 & 0,4 & 1,27 & 244,1 & 1,0 & 1,3 & 245,8 & 1,0 \\
\hline Inseticida (kg) & 0,5 & 113,9 & 0,5 & 0,8 & 172,3 & 0,7 & 1,57 & 336,3 & 1,3 & 1,6 & 336,3 & 1,4 \\
\hline Sementes $(\mathrm{kg})$ & 50,0 & $1.741,7$ & 7,2 & 50,0 & $1.741,7$ & 7,6 & 50 & $1.741,7$ & 6,8 & 50,0 & $1.741,7$ & 7,1 \\
\hline Operações mecanizadas (diesel, L) & 96,8 & $4.619,4$ & 19,1 & 88,6 & $4.227,3$ & 18,4 & 111,24 & $5.309,4$ & 20,8 & 108,0 & $5.152,3$ & 21,1 \\
\hline Mão de obra (hora) & 9,9 & 12,4 & 0,1 & 9,4 & 11,8 & 0,1 & 10,27 & 12,9 & 0,1 & 11,1 & 13,9 & 0,1 \\
\hline Colheita manual (hora) & 0,0 & 0,0 & 0,0 & 0,0 & 0,0 & 0,0 & 64 & 80,4 & 0,3 & 64,0 & 80,4 & 0,3 \\
\hline Colheita mecânica (diesel, L) & 26,7 & $1.274,1$ & 5,3 & 26,7 & $1.274,1$ & 5,5 & 0 & 0,0 & 0,0 & 0,0 & 0,0 & 0,0 \\
\hline Trilha (diesel, L) & 0,0 & 0,0 & 0,0 & 0,0 & 0,0 & 0,0 & 16,43 & 784,2 & 3,1 & 16,4 & 784,0 & 3,2 \\
\hline Irrigação (cm) & 30,0 & $4.961,4$ & 20,5 & 30,0 & $4.961,4$ & 21,6 & 30,0 & $4.961,4$ & 19,4 & 30,0 & $4.961,4$ & 20,3 \\
\hline Monitoramento PI (hora) & 0,0 & 0,0 & 0,0 & 2,0 & 1,9 & 0,0 & 0 & 0,0 & 0,0 & 2,0 & 2,5 & 0,0 \\
\hline $\operatorname{DEE}^{(1)}(\mathrm{MJ})$ & - & 339,9 & 1,4 & - & 293,2 & 1,3 & - & 412,1 & 1,6 & - & 393,4 & 1,6 \\
\hline $\operatorname{Total}^{(2)}\left(\mathrm{MJ} \mathrm{ha}^{-1}\right)$ & - & $24.173,0$ & 100,0 & - & $22.979,3$ & 100,0 & - & $25.518,4$ & 100,0 & - & $24.449,8$ & 100,0 \\
\hline
\end{tabular}

${ }^{(1)} \mathrm{DEE}$, demanda específica de energia do total de horas de cada operação mecanizada, realizada conforme Ulbanere \& Ferreira (1989). (2)Valores totais podem não ser iguais ao somatório, em razão dos arredondamentos independentes.

Tabela 4. Consumo e eficiência (balanço) no uso de energia (MJ), na produção convencional e integrada (PI) de feijão-comum (Phaseolus vulgaris), na terceira época, nas fazendas 3 e 4, em Cristalina, GO.

\begin{tabular}{|c|c|c|c|c|c|c|c|c|c|c|c|c|}
\hline \multirow[t]{3}{*}{ Ingresso } & \multicolumn{6}{|c|}{ Fazenda 3} & \multicolumn{6}{|c|}{ Fazenda 4} \\
\hline & \multicolumn{3}{|c|}{ Convencional } & \multicolumn{3}{|c|}{ PI } & \multicolumn{3}{|c|}{ Convencional } & \multirow[b]{2}{*}{ Quantidade } & \multirow[b]{2}{*}{ Energia } & \multirow[b]{2}{*}{$(\%)$} \\
\hline & Quantidade & Energia & $(\%)$ & Quantidade & Energia & $(\%)$ & Quantidade & Energia & $(\%)$ & & & \\
\hline Calcário (kg) & $1.200,0$ & $1.411,8$ & 6,0 & $1.200,0$ & $1.411,8$ & 6,2 & $1.500,0$ & $1.764,7$ & 7,4 & $1.500,0$ & $1.764,7$ & 7,5 \\
\hline $\mathrm{N}(\mathrm{kg})$ & 100,2 & $6.280,6$ & 26,9 & 103,5 & $6.490,0$ & 28,5 & 89,4 & $5.608,4$ & 23,7 & 89,0 & $5.580,8$ & 23,7 \\
\hline $\mathrm{P}_{2} \mathrm{O}_{5}(\mathrm{~kg})$ & 89,1 & $1.262,4$ & 5,4 & 87,0 & $1.232,6$ & 5,4 & 101,4 & $1.436,6$ & 6,1 & 99,0 & $1.402,6$ & 6,0 \\
\hline $\mathrm{K}_{2} \mathrm{O}(\mathrm{kg})$ & 69,0 & 655,2 & 2,8 & 27,0 & 256,4 & 1,1 & 50,0 & 895,3 & 3,8 & 50,0 & 474,8 & 2,0 \\
\hline Herbicida (kg) & 2,8 & 986,7 & 4,2 & 2,8 & 986,6 & 4,3 & 2,2 & 213,8 & 0,9 & 2,2 & 895,3 & 3,8 \\
\hline Fungicida (kg) & 2,4 & 415,9 & 1,8 & 2,1 & 95,4 & 0,4 & 3,4 & 773,5 & 3,3 & 2,6 & 636,6 & 2,7 \\
\hline Inseticida (kg) & 1,2 & 263,1 & 1,1 & 1,3 & 274,3 & 1,2 & 1,6 & 335,0 & 1,4 & 1,0 & 223,6 & 0,9 \\
\hline Sementes $(\mathrm{kg})$ & 50,0 & $1.741,7$ & 7,5 & 50,0 & $1.741,7$ & 7,7 & 50,0 & $1.741,7$ & 7,3 & 50,0 & $1.741,7$ & 7,4 \\
\hline Operações mecanizadas (diesel, L) & 87,5 & $4.174,5$ & 17,9 & 85,8 & $4.096,1$ & 18,0 & 98,4 & $4.698,0$ & 19,8 & 96,8 & $4.619,6$ & 19,6 \\
\hline Mão de obra (hora) & 9,8 & 12,3 & 0,1 & 9,7 & 12,2 & 0,1 & 10,4 & 13,1 & 0,1 & 10,4 & 13,1 & 0,1 \\
\hline Colheita manual (hora) & 64,0 & 80,4 & 0,3 & 64,0 & 80,4 & 0,4 & 64,0 & 80,4 & 0,3 & 64,0 & 80,4 & 0,3 \\
\hline Colheita mecânica (diesel, L) & 0,0 & 0,0 & 0,0 & 0,0 & 0,0 & 0,0 & 0,0 & 0,0 & 0,0 & 0,0 & 0,0 & 0,0 \\
\hline Recolhe/trilha (diesel, L) & 16,4 & 784,0 & 3,4 & 16,4 & 784,0 & 3,4 & 16,4 & 784,0 & 3,3 & 16,4 & 784,0 & 3,3 \\
\hline Irrigação (cm) & 30,0 & $4.961,4$ & 21,2 & 30,0 & $4.961,4$ & 21,8 & 30,0 & $4.961,4$ & 20,9 & 30,0 & $4.961,4$ & 21,1 \\
\hline Monitoramento PI (hora) & 0,0 & 0,0 & 0,0 & 2,0 & 2,5 & 0,0 & 0,0 & 0,0 & 0,0 & 2,0 & 2,5 & 0,0 \\
\hline $\mathrm{DEE}^{(1)}(\mathrm{MJ})$ & - & 343,9 & 1,5 & - & 334,5 & 1,5 & - & 392,6 & 1,7 & - & 383,2 & 1,6 \\
\hline $\operatorname{Total}^{(2)}\left(\mathrm{MJ} \mathrm{ha}^{-1}\right)$ & - & $23.373,8$ & 100,0 & - & $22.759,9$ & 100,0 & - & $23.698,5$ & 100,0 & - & $23.564,2$ & 100,0 \\
\hline
\end{tabular}

${ }^{(1)} \mathrm{DEE}$, demanda específica de energia do total de horas de cada operação mecanizada, realizada conforme Ulbanere \& Ferreira (1989). ${ }^{(2)}$ Valores totais podem não ser iguais ao somatório, em razão dos arredondamentos independentes. 
de trigo, milho e soja, respectivamente. As maiores economias de energia em PI, observadas por Alluvione et al. (2011), em comparação a Bailey et al. (2003) e ao presente estudo, podem ser atribuídas ao método de análise realizado, ou seja, parcelas experimentais no Norte da Itália e propriedades agrícolas na Grã-Bretanha e no Brasil.

A adubação mineral nitrogenada respondeu pela maior participação no uso de energia, nos dois sistemas de produção, seguida de perto pela irrigação e operações mecanizadas. A participação dos outros insumos no uso total de energia para a produção de feijão, nos dois sistemas de cultivo, foi abaixo de 7,5\%.

Abeliotis et al. (2013) também avaliaram o uso de energia na produção de feijão $P$. coccineus 'GigantesElefantes' e $P$. vulgaris 'Plake', no Norte da Grécia, e constataram que a maior quantidade de energia foi usada pela irrigação, adubação com esterco de ovinos e bovinos, e pelo diesel utilizado na mecanização, que geraram produtividade de $1.150 \mathrm{~kg} \mathrm{ha}^{-1}$ de 'Plake' a $3.130 \mathrm{~kg} \mathrm{ha}^{-1}$ de 'Gigantes-Elefantes'. No presente estudo, o consumo de energia pela irrigação, foi, no entanto, similar para ambos os sistemas de produção de feijão. Comparado ao sistema convencional, o PI teve sensível diminuição do uso de adubo nitrogenado (14,8\% de redução), adubo potássico (redução de até 60,9\%), inseticida (redução de até 60\%) e fungicida (redução de até 66,7\%). Embora alguns insumos apresentem alta demanda energética em sua fabricação - como adubo mineral nitrogenado e óleo diesel -, eles são utilizados em grande quantidade que, se diminuída pelo uso de bactérias fixadoras de nitrogênio ou pela adoção de controle de tráfego na lavoura, poderiam contribuir sensivelmente para a diminuição do uso de energia na produção de feijão (Crews \& Peoples, 2004; Gasso et al., 2013).

Os resultados de uso de energia por hectare, embora úteis para mostrar a magnitude do uso total de energia para a produção e para identificar os insumos que mais participam da produção de grãos numa fazenda, eles não consideram a produtividade das culturas. A medição da eficiência energética, entretanto, leva em consideração a produtividade de grãos e, assim, conforme Bailey et al. (2003), é bastante apropriada para investigar se a variação do uso de energia é refletida na produtividade. No presente estudo, a eficiência energética do PI foi 7,5\% superior à observada no convencional (Figura 2). Bailey et al. (2003) também observaram pouca diferença entre o
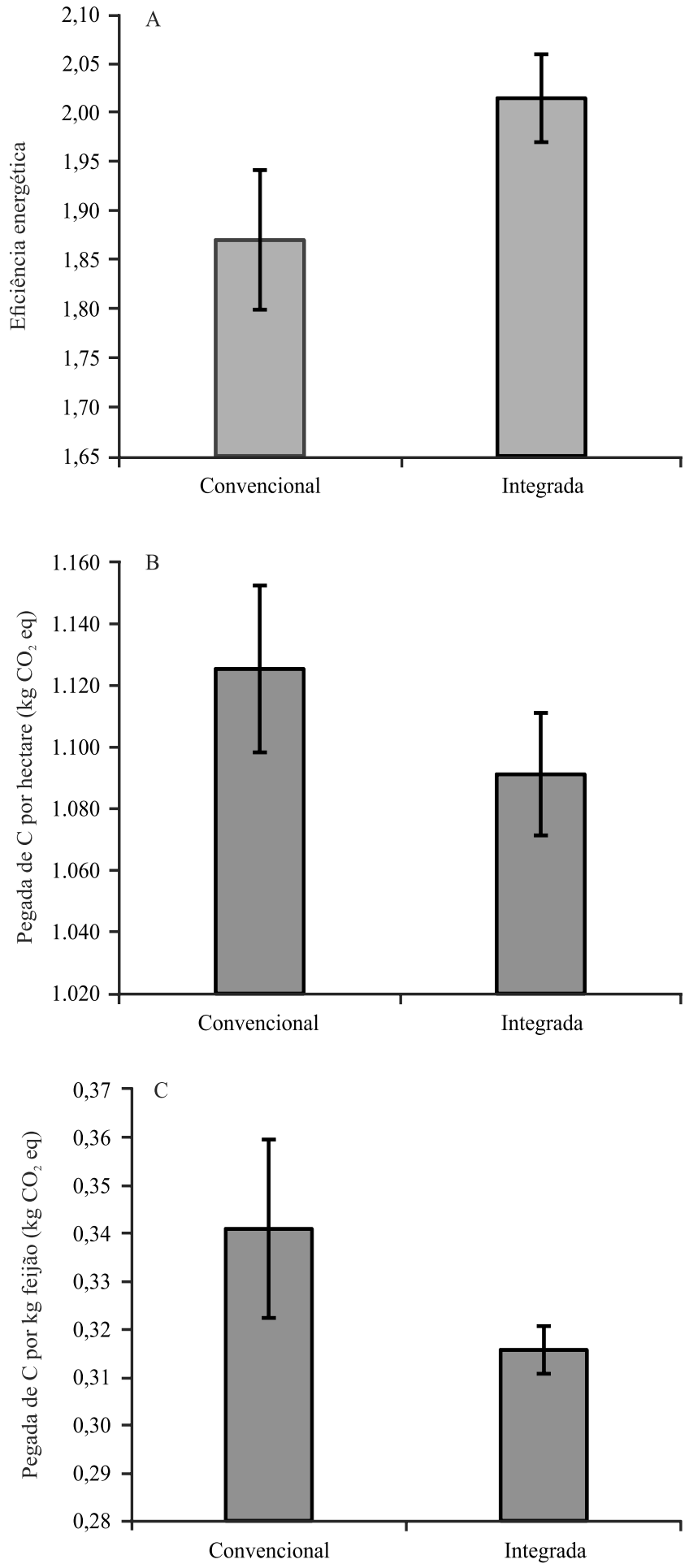

Figura 2. Eficiência energética (A) e pegada de carbono por hectare (B) e por quilograma de feijão-comum $(\mathrm{C})$, nos sistemas de produção convencional e integrada, na terceira época, na região de Cristalina, GO. Os valores no centro da coluna indicam as médias $(n=4)$ de cada variável. As barras indicam o erro padrão da média. 
sistema de produção convencional e o sistema PI, na Grã-Bretanha, e enfatizaram que há espaço para adoção de outros manejos conservacionistas, especialmente o plantio direto ou o cultivo mínimo, nas propriedades com cultivo PI. No presente estudo, as fazendas adotavam o plantio direto, apesar do limitado uso de plantas de cobertura na rotação. A palhada gerada pelas plantas de cobertura pode contribuir com a preservação da umidade do solo, o que implica menor número de irrigações (Moreira et al., 1999), e contribuir com a supressão da infestação de plantas daninhas (Oliveira Jr. et al., 2014), o que pode levar ao menor uso de herbicidas. Essas contribuições também podem levar a um menor consumo de energia, sem comprometer com a produtividade da cultura. A eficiência energética em PI é similar à obtida pelo cultivo de canola (Brassica napus) e soja (Glycine max Merr.) no Nordeste dos EUA (Camargo et al., 2013), mas, comparações com outras culturas devem ser feitas com cautela, pois a canola produz grande quantidade de óleo por hectare, e isto exige grande quantidade de fertilizante mineral nitrogenado, para proporcionar mais energia para a produção, enquanto a soja, uma leguminosa que fixa nitrogênio atmosférico, demanda menos energia.

Quanto à pegada de carbono por hectare, a adoção do sistema PI, para a produção de feijão, não resultou em diminuição significativa em relação à produção convencional (Figura 2). Contudo, a pegada de carbono, por quilograma de feijão produzido em PI, foi significativamente menor $(7,4 \%)$ do que em convencional. As pequenas diferenças entre os dois tipos de cultivo, para as variáveis analisadas, também

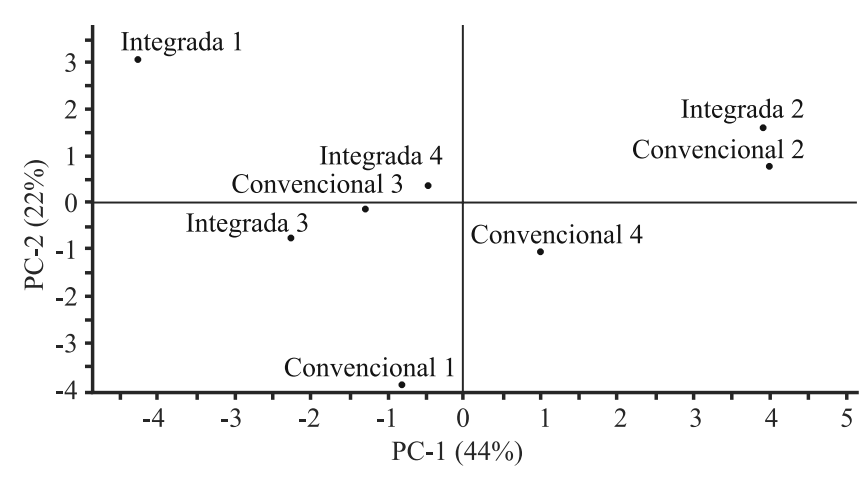

Figura 3. Distribuição das fazendas $(n=4)$ com produção convencional e produção integrada de feijão-comum, para as componentes principais 1 (PC-1) e 2 (PC-2), em que se consideram as pegadas de carbono por hectare e por quilograma de grão. se refletiram na análise de componentes principais (Figura 3), em que há uma tênue separação entre as fazendas com produção convencional e com produção integrada quanto à componente principal 2, com destaque para o uso de fertilizantes minerais. Em estudo realizado em 24 propriedades, em sistema convencional, e 15 com produção integrada, Hillier et al. (2009) também não encontraram diferença significativa para a pegada de carbono por hectare de diferentes cultivos, no Leste da Escócia. No entanto, Abeliotis et al. (2013) constataram, em relação ao cultivo convencional de feijão no Norte da Grécia, que a produção integrada resultou em diminuição mais pronunciada (cerca de $61 \%$ ) da pegada de carbono por quilograma de feijão produzido. As magnitudes diferentes entre o presente estudo e o realizado por Abeliotis et al. (2013) são, provavelmente, decorrentes da menor área de trabalho conduzida na Grécia (entre 2,6 e 22,0 ha). A inexistência de diferença entre os sistemas PI e convencional, para a pegada de carbono por hectare, e as diferenças pouco pronunciadas de pegada de carbono por quilograma de feijão estão coerentes com a análise econômica da produção de feijão, realizada por Silva et al. (2012), nos dois sistemas de cultivo, nos mesmos locais, em que observaram que PI proporcionou uma receita líquida operacional superior à obtida com o sistema convencional, em metade das fazendas avaliadas.

Pelos dados observados na presente avaliação e por Silva et al. (2012), há margem para o aperfeiçoamento das práticas agronômicas adotadas em PI, para a busca de melhores resultados quanto ao impacto ambiental, indicados pelo uso e eficiência de energia, pegada de carbono por hectare e por quilograma do produto.

\section{Conclusões}

1. O sistema de produção integrada de feijão-comum irrigado consome menos energia e apresenta eficiência energética superior ao sistema de produção convencional.

2. Em comparação com a produção convencional, o benefício ambiental da produção de feijão, no sistema de produção integrada, é significativo apenas para a pegada de carbono por quilograma de feijão.

3. A maior eficiência no manejo da adubação nitrogenada, especialmente com o uso de inoculantes, pode favorecer sensivelmente o balanço energético e a pegada de carbono por quilograma de feijão produzido sob irrigação.

Pesq. agropec. bras., Brasília, v.51, n.9, p.1069-1077, set. 2016 DOI: $10.1590 / \mathrm{S} 0100-204 \mathrm{X} 2016000900006$ 


\section{Agradecimentos}

À Cooperativa Coacris e à empresa Brava Agronegócios, pelo apoio na realização do levantamento de dados; aos produtores Edilso Danielle, Ivan Gasparetto, Marcelino Sato e Renato Zóboli, pelo tempo dedicado a entrevistas e disponibilização de área para o estudo.

\section{Referências}

ABELIOTIS, K.; DETSIS, V.; PAPPIA, C. Life cycle assessment of bean production in the Prespa National Park, Greece. Journal of Cleaner Production, v.41, p.89-96, 2013. DOI: 10.1016/j. jclepro.2012.09.032.

ALlUVIONE, F.; MORETTI, B.; SACCO, D.; GRIGNANI, C. EUE (energy use efficiency) of cropping systems for a sustainable agriculture. Energy, v.36, p.4468-4481, 2011. DOI: 10.1016/j. energy.2011.03.075.

AUDSLEY, E.; STACEY, K.; PARSONS, D.J.; WILLIAMS, A.G. Estimation of the greenhouse gas emissions from agricultural pesticide manufacture and use. Cranfield: Cranfield University, 2009. 24p.

BAILEY, A.P.; BASFORD, W.D.; PENLINGTON, N.; PARK, J.R.; KEATINGE, J.D.H.; REHMAN, T.; TRANTER, R.B.; YATES, C.M. A comparison of energy use in conventional and integrated arable farming systems in the UK. Agriculture, Ecosystems and Environment, v.97, p.241-253, 2003. DOI: 10.1016/S01678809(03)00115-4.

BASSINELLO, P.Z. Qualidade nutricional, funcional e tecnológica do feijão. In: KLUTHCOUSKI, J.; STONE, L.F.; AIDAR, H. (Ed.). Fundamentos para uma agricultura sustentável, com ênfase na cultura do feijoeiro. Santo Antônio de Goiás: Embrapa Arroz e Feijão, 2009. p.385-424.

BOLLER, E.F.; AVILLA, J.; GENDRIER, J.P.; JÖRG, E.; MALAVOLTA, C. (ed.). Integrated production in Europe: 20 years after the declaration of Ovronnaz. Waedenswil: International Organization for Biological and Integrated Control, 1998. 41p. (IOBC-WPRS Bulletin, 21).

CAMARGO, G.G.T.; RYAN, M.R.; RICHARD, T.L. Energy use and greenhouse gas emissions from crop production using the farm energy analysis tool. BioScience, v.63, p.263-273, 2013. DOI: 10.1525/bio.2013.63.4.6.

COWELL, S.J.; PARKINSON, S. Localization of UK food production: an analysis using land area and energy as indicators. Agricultural, Ecosystems and Environment, v.94, p.221-236, 2003. DOI: 10.1016/S0167-8809(02)00024-5.

CREWS, T.E.; PEOPLES, M.B. Legume versus fertilizer sources of nitrogen: ecological tradeoffs and human needs. Agriculture, Ecosystems and Environment, v.102, p.279-297, 2004. DOI: 10.1016/j.agee.2003.09.018.

GASSO, V.; SØRENSEN, C.A. G.; OUDSHOORN, F.W.; GREEN, O. Controlled traffic farming: a review of the environmental impacts. European Journal of Agronomy, v.48, p.66-73, 2013. DOI: 10.1016/j.eja.2013.02.002.

GRISSO, R.B.; PERUMPRAL, J.V.; ROBERSON, G.T.; PITMAN, R. Predicting tractor diesel fuel consumption. Petersburg: Virginia Cooperative Extension: College of Agriculture and Life Sciences: Virginia Polytechnic Institute: Virginia State University, 2010. 10p. (Publication 442-073).

HELANDER, C.A.; DELIN, K. Evaluation of farming systems according to valuation indices developed within a European network on integrated and ecological arable farming systems. European Journal of Agronomy, v.21, p.53-67, 2004. DOI: 10.1016/S1161-0301(03)00089-3.

HILlIER, J.; HAWES, C.; SQUIRE, G.; HILTON, A.; WALE, S.; SMITH, P. The carbon footprints of food crop production. International Journal of Agricultural Sustainability, v.7, p.107118, 2009. DOI: 10.3763/ijas.2009.0419.

MAROUELLI, W.A.; SILVA, W.L.C. Seleção de sistemas de irrigação para hortaliças. Brasília: Embrapa Hortaliças, 2011. 22p. (Embrapa Hortaliças. Circular técnica, 98).

MELLO, R. de. Um modelo para análise energética de agroecossistemas. Revista de Administração de Empresas, v.29, p.45-61, 1989. DOI: 10.1590/S0034-75901989000400005.

MOREIRA, J.A.A.; STONE, L.F.; PEREIRA, A.L. Manejo da irrigação do feijoeiro em plantio direto: cobertura do solo. Santo Antônio de Goiás: Embrapa Arroz e Feijão, 1999. 2p. (Embrapa Arroz e Feijão. Pesquisa em foco, 26).

MORRIS, C.; WINTER, M. Integrated farming systems: the third way for European agriculture? Land Use Policy, v.16, p.193-205, 1999. DOI: 10.1016/S0264-8377(99)00020-4.

OLIVEIRA, JR., R.S.; RIOS, F.A.; CONSTANTIN, J.; ISHIIWAMOTO, E.L.; GEMELLI, A.; MARTINI, P.E. Grass straw mulching to suppress emergence and early growth of weeds. Planta Daninha, v.32, p.11-17, 2014. DOI: 10.1590/S010083582014000100002 .

OLIVEIRA, J.S.; ZOCOLER, J.L. Custos da irrigação e receita líquida do feijoeiro num sistema pivô central com bombeamento a diesel sob variação do comprimento da tubulação de recalque e desnível topográfico. Pesquisa Aplicada e Agrotecnologia, v.3, p.7-14, 2010.

PASSUELLO, A.C.B.; OLIVEIRA, A.F. de; COSTA, E.B. da; KIRCHHEIM, A.P. Aplicação da avaliação do ciclo de vida na análise de impactos ambientais de materiais de construção inovadores: estudo de caso da pegada de carbono de clínqueres alternativos. Ambiente Construído, v.14, p.7-20, 2014. DOI: 10.1590/S1678-86212014000400002.

PAULA JÚNIOR, T.J.; VIEIRA, R.F.; TEIXEIRA, H.; COELHO, R.R.; CARNEIRO, J.E.S.; ANDRADE, M.J.B.; RESENDE, A.M. Informações técnicas para o cultivo de feijoeiro-comum na região central brasileira: 2007-2009. Viçosa: EPAMIG, 2008. $180 \mathrm{p}$.

PIMENTEL, D.; PATZEK, T.W. Ethanol production using corn, switchgrass, and wood; biodiesel production using soybean and sunflower. Natural Resources Research, v.14, p.65-76, 2005. DOI: $10.1007 / \mathrm{s} 11053-005-4679-8$. 
PIMENTEL, D.; PIMENTEL, M. Global environmental resources versus world population growth. Ecological Economics, v.59, p.195-198, 2006. DOI: 10.1016/j.ecolecon.2005.11.034.

PORTOCARRERO, M.A.; KOSOSKI, A.R. Alimentos seguros: uma política de governo. In: ZAMBOLIM, L.; NASSER, L.C.B.; ANDRIGUETO, J.R.; TEIXEIRA, J.M.A.; KOSOSKI, A.R.; FACHINEllO, J.C. (Org.). Produção integrada no Brasil: agropecuária sustentável alimentos seguros. Brasília: MAPA, 2009. p.13-29.

QUADROS, K.R. de; KOKUSZKA, R. Balanço energético em sistemas de produção convencional e agroecológico de feijão, na região de Rebouças-PR. Revista Brasileira de Agroecologia, v.2, p.50-54, 2007.

RAUCCI, G.S.; MOREIRA, C.S.; ALVES, P.A.; MELLO, F.F.C.; FRAZÃO, L. de A.; CERRI, C.E.C.; CERRI, C.C. Greenhouse gas assessment of Brazilian soybean production: a case study of Mato Grosso state. Journal of Cleaner Production, v.96, p.418-425, 2015. DOI: 10.1016/j.jclepro.2014.02.064.

ROTZ, C.A.; MONTES, F.; CHIANESE, D.S. The carbon footprint of dairy production systems through partial life cycle assessment. Journal of Dairy Science, v.93, p.1266-1282, 2010. DOI: $10.3168 /$ jds.2009-2162.

SÁ, J.M. e; URQUIAGA, S.; JANTALIA, C.P.; SOARES, L.H. de B.; ALVES, B.J.R.; BODDEY, R.M.; MARCHÃO, R.L.; VILELA, L. Balanço energético da produção de grãos, carne e biocombustíveis em sistemas especializados e mistos. Pesquisa Agropecuária Brasileira, v.48, p.1323-1331, 2013. DOI: 10.1590/ S0100-204X2013001000003.

SILVEIRA, P.M. da; RAMOS, M.M.; OLIVEIRA, R.A. de. Manejo da irrigação do feijoeiro com o uso do irrigâmetro. Santo Antônio de Goiás: Embrapa Arroz e Feijão, 2009. 4p. (Embrapa Arroz e Feijão. Circular técnica, 84).

SILVA, A.G.; WANDER, A.E.; BARBOSA, F.R.; GONZAGA, A.C. de O.; SILVA, J.G. da. Análise econômica da produção de feijão comum em sistema de produção convencional e de produção integrada, em Cristalina, Estado de Goiás, e Unaí, Estado de Minas Gerais, maio de 2009 a abril de 2010. Informações Econômicas, v.42, p.55-64, 2012.

SOARES, L.H. de B.; ALVES, B.J.R.; URQUIAGA, S.; BODDEY, R.M. Mitigação das emissões de gases efeito estufa pelo uso de etanol da cana-de-açúcar produzido no Brasil. Seropédica: Embrapa Agrobiologia, 2009. 14p. (Embrapa Agrobiologia. Circular técnica, 27).

SOLOMON, S.; QIN, D.; MANNING, M.; CHEN, Z.; MARQUIS, M.; AVERYT, K.; TIGNOR, M.M.B.; MILLER JR., H.L.; CHEN, Z. (Ed.). Climate Change 2007: the physical science basis. Cambridge: Cambridge University Press, 2007. 996p. Contribution of Working Group I to the Fourth Assessment Report of the Intergovernmental Panel on Climate Change.

SOUZA, J.L. de; CASALI, V.W.D.; SANTOS, R.H.S.; CECON, P.R. Balanço e análise da sustentabilidade energética na produção orgânica de hortaliças. Horticultura Brasileira, v.26, p.433-440, 2008. DOI: 10.1590/S0102-05362008000400003.

ULBANERE, R.C.; FERREIRA, W.A. Análise do balanço energético para a produção de milho no Estado de São Paulo. Energia na Agricultura, v.4, p.35-42, 1989.

WEST, T.O.; MARLAND, G. A synthesis of carbon sequestration, carbon emissions, and net carbon flux in agriculture: comparing tillage practices in the United States. Agriculture, Ecosystems and Environment, v.91, p.217-232, 2002. DOI: 10.1016/S01678809(01)00233-X.

WIEDMANN, T.; MINX, J. A definition of 'carbon footprint'. In: PERTSOVA, C.C. (Ed.). Ecological Economics Research Trends. New York: Nova Science, 2007. p.1-11. (ISAUK Research Report 07-01).

WILLIAMS, A.G.; AUDSLEY, E.; SANDARS, D.L. Determining the environmental burdens and resource use in the production of agricultural and horticultural commodities: Defra Research Project IS0205. Bedford: Cranfield University and Defra, 2006. 96p.

$\overline{\text { Recebido em } 31 \text { de agosto de } 2015 \text { e aprovado em } 14 \text { de março de } 2016}$ 